\title{
Anti-metastatic effect of Smilax china L. extract on MDA-MB-231 cells
}

\author{
KYOUNG JIN NHO, JIN MI CHUN and HO KYOUNG KIM \\ Herbal Medicine Resources Group, Korea Institute of Oriental Medicine, Daejeon 305-811, Republic of Korea
}

Received August 23, 2013; Accepted March 17, 2014

DOI: $10.3892 / \mathrm{mmr} .2014 .2698$

\begin{abstract}
Cancer metastases are not always cured by chemotherapy. Conventional and alternative drugs, including Chinese herbal remedies, have been developed to target metastatic cancer cells. Smilax china L. (SCL), a member of the Smilacaceae family, exerts anti-inflammatory, detoxification and anti-cancer effects. However, the effect of SCL on breast cancer cell metastasis and the underlying mechanisms are yet to be elucidated. The aim of this study was to investigate the effect of a SCL ethanol extract (SCLE) on the proliferation and migration of MDA-MB-231 human breast cancer cells, as well as the expression of urokinase plasminogen activator (uPA), uPA receptor (UPAR) and tissue inhibitors of metalloproteinases (TIMPs). Cell proliferation was assessed using the Cell Counting Kit- 8 and cell migration was determined by wound healing assay. Quantitative polymerase chain reaction was performed to quantify the mRNA levels of UPA, uPAR and TIMPs. SCLE markedly inhibited the proliferation and migration of MDA-MB-231 cells, and reduced the mRNA levels of the extracellular matrix (ECM) degradation-associated molecules uPA, uPAR. By contrast, SCLE significantly increased the mRNA levels of TIMP1 and TIMP2. These findings show that SCLE exerts an anti-metastatic effect on human breast cancer cells, which may involve the modulation of ECM degradation.
\end{abstract}

\section{Introduction}

Breast cancer is the second leading cause of cancer-related mortality among females worldwide. Although improved screening techniques have aided the detection of breast cancer at the early stages and advanced treatments have markedly improved patient survival, tumor invasion and metastasis still

Correspondence to: Dr Ho Kyoung Kim, Herbal Medicine Resources Group, Korea Institute of Oriental Medicine, 1672 Yuseong-daero, Yuseong-gu, Daejeon 305-811, Republic of Korea

E-mail: hkkim@kiom.re.kr

Key words: Smilax china L., invasion, urokinase plasminogen activator, tissue inhibitors of metalloproteinases, MDA-MB-231 contribute to the majority of breast cancer-associated mortalities (1).

Metastasis is a series of events that involves the detachment of tumor cells from the primary tumor site, their adhesion, migration and invasion into blood or lymphatic vessels and their interaction with target tissues. The invasion of tumor cells into target tissues results in the formation of secondary tumors $(2,3)$. These events occur repeatedly during tumor invasion, and perturbation of the adhesiveness and motility of tumor cells and their invasion into target tissues has been proposed as a method of preventing cancer progression $(4,5)$.

Plants are valuable sources of natural therapeutic compounds. Research into oriental medicines has increased over the past decade, with the aim of identifying novel drugs, including anti-cancer agents (6-8). Several herb-based constituents and extracts have been found to reduce tumor growth and inhibit the metastasis of human cancer cells in in vitro and in vivo models $(9,10)$. Therefore, such compounds may be valuable tools in cancer therapy.

Smilax china L. (SCL), a member of the Smilacaceae family, is used in Traditional Chinese Medicine as a diuretic and detoxification agent and to treat various conditions, including rheumatic arthritis, lumbago, gout, tumors and inflammatory diseases (11). Pharmacological investigations indicate that SCL exhibits anti-tumor (12) and anti-inflammatory (13) effects. However, the effect of SCL ethanol extract (SCLE) treatment on the highly metastatic MDA-MB-231 human breast cancer cell line is yet to be elucidated. Therefore, in the present study, the anti-metastatic effect of SCLE on MDA-MB-231 cells was investigated.

\section{Materials and methods}

Materials. All plastic materials were purchased from Falcon Labware (Becton Dickinson and Company, Franklin Lakes, NJ, USA). Fetal bovine serum (FBS), phosphate-buffered saline and penicillin G/streptomycin were obtained from Gibco-BRL (Grand Island, NY, USA). RPMI-1640 medium was purchased from Welgene, Inc. (Daegu, Korea). The Cell Counting Kit-8 (CCK-8) was obtained from Dojindo Molecular Technologies, Inc. (Gaithersburg, MD, USA).

Preparation of plant extracts. SCL was purchased as a dried herb from Omniherb Co. (Yeoungcheon, Korea). The dried bark of SCL (200 g) was extracted twice using $70 \%$ ethanol 
Table I. Primer sequences used in quantitative polymerase chain reaction analysis.

\begin{tabular}{lllcc}
\hline Genes & \multicolumn{1}{c}{ Forward } & \multicolumn{1}{c}{ Reverse } & Accession number & Length (bp) \\
\hline uPA & ACCCAAAGAAGGAGGACTAC & GTGTAGGATGAGGTTTTCCA & NM_002658 & 101 \\
uPAR & GGTTGTGTGTGGGTTAGACT & CTCTCACAGCTCATGTCTGA & NM_002659 & 117 \\
TIMP1 & TGGACTCTTGCACATCACTA & GATGGATAAACAGGGAAACA & NM_003254 & 133 \\
TIMP2 & GCTCTGTTGATTTTGTTCC & CTGCTTGTCAACTTTCAACA & NM_003255 & 125 \\
GAPDH & TCAAGCTCATTTCCTGGTAT & GTGAGGGTCTCTCTCTTCCT & NM_002046 & 141 \\
\hline
\end{tabular}

$\mathrm{uPA}$, urokinase plasminogen activator; uPAR, uPA receptor; TIMP, tissue inhibitor of metalloproteinase.

with $2 \mathrm{~h}$ reflux, and the extract was concentrated under reduced pressure. The decoction was filtered, lyophilized and stored at $4^{\circ} \mathrm{C}$. The yield of dried extract from the starting crude material was $\sim 12.7 \%$ (w/w). The SCLE was diluted in culture media to the final concentrations indicated for each experiment.

Cell culture. MDA-MB-231 cells were obtained from the American Type Culture Collection (Rockville, MD, USA) and cultured in RPMI-1640 medium supplemented with $10 \%$ FBS, $100 \mathrm{U} / \mathrm{ml}$ penicillin and $100 \mu \mathrm{g} / \mathrm{ml}$ streptomycin. The culture was performed at $37^{\circ} \mathrm{C}$ in $5 \% \mathrm{CO}_{2}$. Subsequent to reaching $80 \%$ confluence, the cells were detached using $0.25 \%$ trypsin-EDTA. Cells were then subcultured further.

Cell viability assay. Water-soluble tetrazolium salt- 8 , which is reduced to yellow water-soluble formazan by the dehydrogenase released from mitochondria, was the effective constituent of the CCK-8. Absorbance was measured at $450 \mathrm{~nm}$ using a Benchmark Plus Microplate Spectrophotometer (Bio-Rad, Hercules, CA, USA). Cytotoxicity was expressed as a percentage of the absorbance measured in the control and SCLE-treated cells.

Wound healing assay. MDA-MB-231 cells were plated in a 12 -well plate at a concentration of $5 \times 10^{5}$ cells/well and allowed to form a confluent monolayer for $24 \mathrm{~h}$. Cells were wounded using a yellow pipette tip and cellular debris was removed by washing with RPMI-1640 medium. The wounded monolayer was incubated in the presence or absence of SCLE (25 and $50 \mu \mathrm{g} / \mathrm{ml}$ ) for $24 \mathrm{~h}$ in either RPMI-1640 plus $10 \% \mathrm{FBS}$ as a positive control or RPMI-1640 plus $1 \%$ FBS (CON). Images were captured of the cell migration into the wounded area using phase-contrast microscopy.

Quantitative polymerase chain reaction ( $q P C R)$. qPCR was performed using the Rotor-Gene 3000 (Corbett Research, Sydney, Australia) system using SYBR ${ }^{\circledR}$ Green Master Mix (Qiagen, Tokyo, Japan). The percentage of target gene expression relative to the control was normalized using the GAPDH internal control according to the $2^{-\Delta \Delta C t}$ analysis. Primers for the target genes were designed using Primer3 software (Table I) (14).

Statistical analysis. Statistical analyses were performed using the Prism 5 software (GraphPad Software, Inc., San Diego, CA, USA). Analysis of variance followed by a Dunett's test were performed. A value of $\mathrm{P}<0.05$ was considered to indicate a statistically significant difference.

\section{Results}

Effect of SCLE on MDA-MB-231 cell viability. The cytotoxic effect of SCLE was investigated in three cancer cell lines (HepG2, A549 and MDA-MB-231) using the CCK-8. SCLE was observed to reduce the viability of each of these cell lines in a concentration-dependent manner (Fig. 1A). However, SCLE was found to have a weaker effect on the viability of A549 and MDA-MB-231 cells than that of HepG2 cells. SCLE was observed to reduce the viability of MDA-MB-231 cells from 100 to $60 \%$ in a concentration- and time-dependent manner (Fig. 1B). The viability of MDA-MB-231 cells was significantly reduced following treatment with $100-400 \mu \mathrm{g} / \mathrm{ml}$ SCLE for 12 or $24 \mathrm{~h}(\mathrm{P}<0.01)$ (Fig. 1B). Based on these results, non-cytotoxic concentrations of SCLE (25 and $50 \mu \mathrm{g} / \mathrm{ml})$ were used in further experiments.

Effect of SCLE on the metastatic potential of MDA-MB-231 cells. Due to the highly invasive nature of triple-negative breast cancers, the present study investigated the effect of SCLE on the invasive potential of MDA-MB-231 cells in vitro. A wound healing assay was performed to determine the effect of SCLE on the inhibition of MDA-MB-231 cell migration. Twenty-four hours after cell monolayers were wounded, control cell monolayers (RPMI-1640 plus 1\% FBS) had completely filled the wounded area. SCLE treatment was observed to inhibit the migration of MDA-MB-231 cells in a concentration-dependent manner (Fig. 2A). Densitometric analysis showed that treatment with $50 \mu \mathrm{g} / \mathrm{ml} \mathrm{SCLE} \mathrm{reduced}$ the migration of MDA-MB-231 cells by $\sim 50 \%$ (Fig. 2B). These results suggest that SCLE effectively inhibits the migration of the highly invasive MDA-MB-231 breast cancer cell line.

Effect of SCLE on the expression of extracellular matrix (ECM) degradation-associated molecules. The overexpression and activation of urokinase plasminogen activator (UPA) and the uPA receptor (UPAR) play an important role in the invasion of breast cancer cells by stimulating ECM degradation and thereby promoting cell migration. Therefore, the present study aimed to investigate whether the SCLE-induced inhibition of migration in MDA-MB-231 cells correlated with modulation of uPA and UPAR. SCLE treatment was found to reduce the mRNA levels of UPA and uPAR in a concentration-dependent manner (Fig. 3). By contrast, the mRNA levels of tissue inhibitor of metalloproteinase (TIMP) 1 and 2 were significantly increased following SLCE treatment $(\mathrm{P}<0.05)$. These data suggest that the 
A

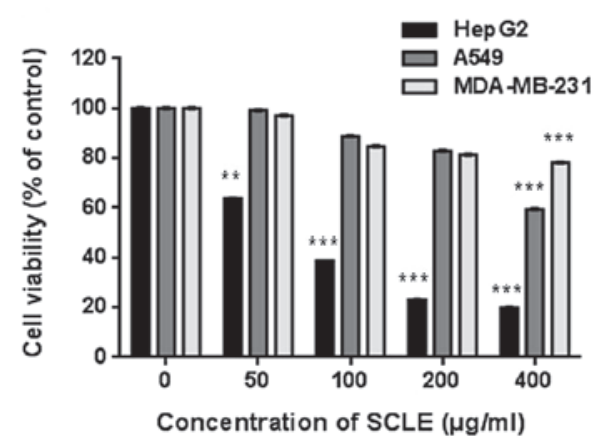

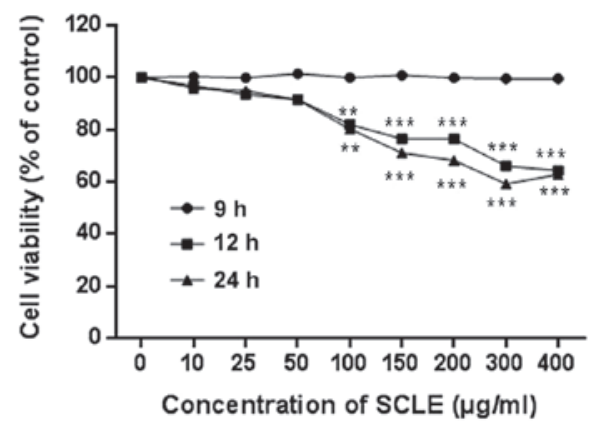

Figure 1. SCLE treatment reduces MDA-MB-231 cell viability. (A) HepG2, A549 and MDA-MB-21 cells were treated with various concentrations of SCLE for 24 h. (B) MDA-MB-231 cells were treated with various concentrations of SCLE for 9, 12 and $24 \mathrm{~h}$. Cell viability was then estimated using the Cell Counting Kit-8. Data are presented as the mean \pm standard deviation of triplicate samples. ${ }^{* *} \mathrm{P}<0.01$ and ${ }^{* * *} \mathrm{P}<0.001$ vs. untreated cells. SCLE, Smilax china L ethanol extract.

A

MDA-MB-231

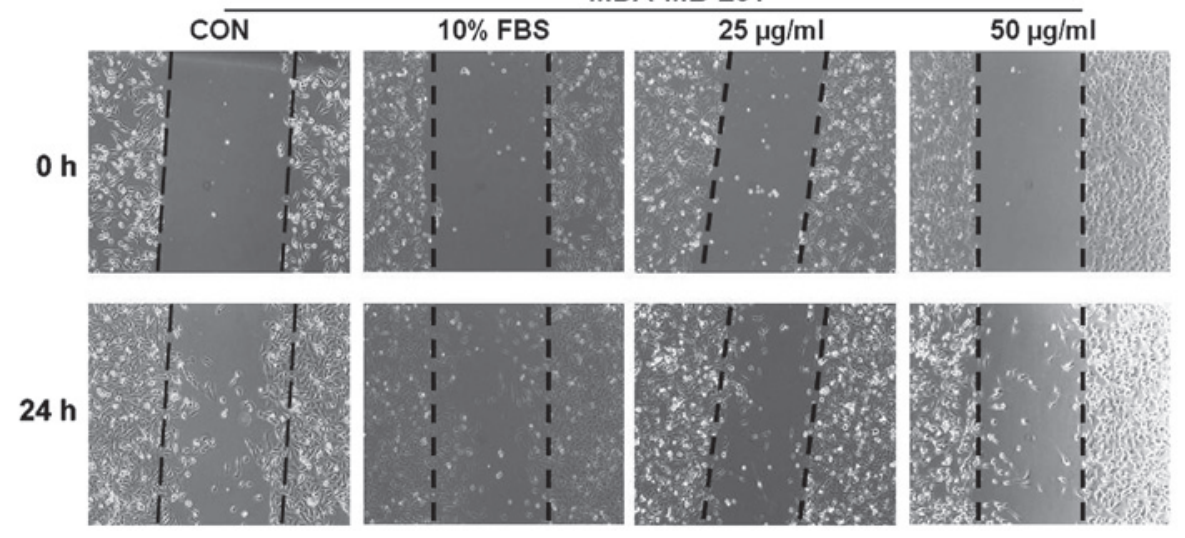

B

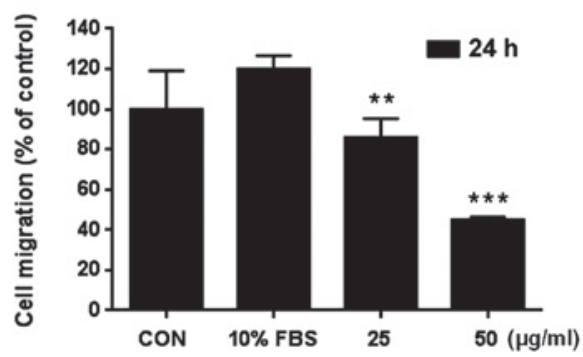

Figure 2. SCLE treatment reduces MDA-MB-231 cell migration. MDA-MB-231 cells were treated with $25 \mathrm{or} 50 \mu \mathrm{g} / \mathrm{ml} \mathrm{SCLE}$ for $24 \mathrm{~h}$. Alternatively, cells were treated with $10 \%$ FBS as a positive control or with $1 \%$ FBS (CON). (A) Cell migration into the wounded area (indicated by dashed lines) was observed using an inverted phase contrast microscope (magnification, x200). (B) Quantitative assessment of the number of cells in the wounded area is expressed as the mean \pm standard deviation of three independent experiments. ${ }^{* *} \mathrm{P}<0.01$ and ${ }^{* * *} \mathrm{P}<0.001$ vs. CON cells. SCLE, Smilax china $\mathrm{L}$ ethanol extract; FBS, fetal bovine serum.

A

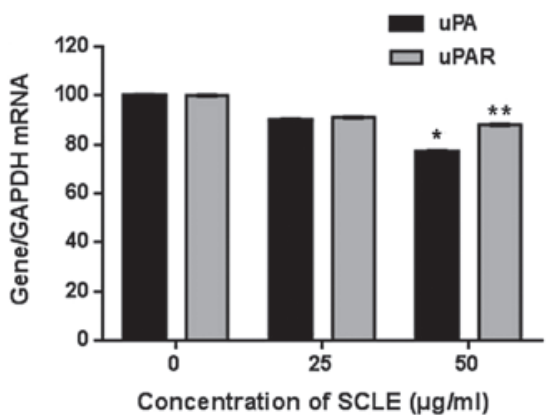

B

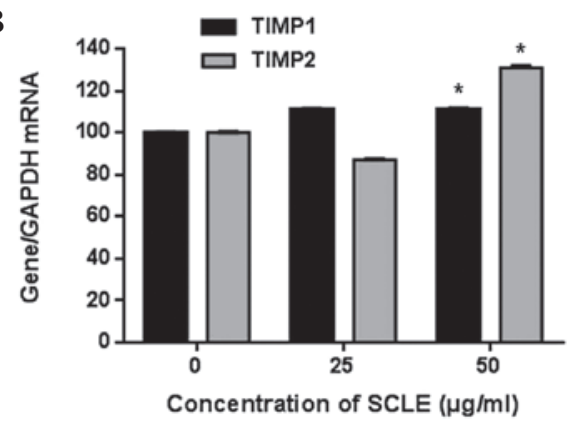

Figure 3. SCLE treatment alters the mRNA levels of extracellular matrix degradation-associated molecules in MDA-MB-231 cells. (A and B) Cells were treated with various concentrations of SCLE for $24 \mathrm{~h}$. uPA, uPAR TIMP1 and TIMP2 mRNA levels were determined using quantitative polymerase chain reaction. Data are expressed as the mean \pm standard deviation of triplicate samples. ${ }^{*} \mathrm{P}<0.05$ and ${ }^{* *} \mathrm{P}<0.01$ vs. untreated cells. uPA, urokinase plasminogen activator; uPAR, uPA receptor; TIMP, tissue inhibitor of metalloproteinase; SCLE, Smilax china L ethanol extract. 
SCLE-mediated inhibition of MDA-MB-231 cell migration and metastasis may be dependent on the degradation of the ECM.

\section{Discussion}

In patients with cancer, mortality is rarely a direct consequence of the growth of the primary tumor, but of primary tumor metastasis. Therefore, the prevention and suppression of tumor invasion and metastasis may be a promising strategy for improving the survival of patients with malignant tumors. The present study aimed to investigate the anti-metastatic effects of SCLE, a Chinese herbal medicine, and the mechanisms underlying these effects. SCLE was found to have an anti-metastatic effect on human breast cancer cells. SCLE reduced MDA-MB-21 cell migration in a concentration-dependent manner in vitro (Fig. 2), indicating that SCLE has potent anti-metastatic activity in vitro.

Metastasis is the major cause of mortality in patients with cancer, and is a multifaceted process that results from coordinated events, including cancer cell invasion, migration and adhesion (15). Proteolytic enzyme-induced degradation of the ECM and basement membrane and the subsequent invasion of cancerous cells are essential early steps in metastasis (16). uPA is an important proteolytic enzyme that degrades the ECM, and the expression of UPA and UPAR is associated with increased tumor cell invasion and metastasis in breast cancer $(17,18)$. It is well established that overexpression of uPA in breast cancer is a strong indicator of poor prognosis. UPA binds UPAR on the cell membrane and subsequently degrades cell surface-associated plasminogen (19). Therefore, the present study investigated whether the anti-invasive effect of SCLE in MDA-MB-21 cells correlated with the inhibition of UPA and UPAR activity. SCLE treatment was observed to reduce the mRNA levels of UPA and uPAR (Fig. 3A). Matrix metalloproteinases are a large group of proteolytic enzymes that are involved in degrading and remodeling the ECM in response to a number of pathological stimuli (20). These enzymes are controlled by their endogenous inhibitors TIMP1 and TIMP2 (21). Therefore, increased TIMP activity can inhibit cancer cell invasion and metastasis $(22,23)$. SCLE treatment increased the mRNA levels of TIMP1 and TIMP2 in MDA-MB-231 cells (Fig. 3B). These effects correlated with the inhibition of MDA-MB-231 cell invasion by SCLE.

The present study has demonstrated that SCLE effectively suppresses invasiveness in the MDA-MB-231 human breast cancer cell line. The mechanism underlying this effect may involve modulation of UPA, uPAR and TIMP expression. The results of this study indicate that SCLE may be a potential agent for the treatment of cancer metastasis.

\section{Acknowledgements}

This study was supported by the Construction of the Basis for Practical Application of Herbal Resources (no. K12020) from the Korea Institute of Oriental Medicine.

\section{References}

1. Kundu N, Campbell P, Hampton B, Lin CY, Ma X, Ambulos N, Zhao XF, Goloubeva O, Holt D and Fulton AM: Antimetastatic activity isolated from Colocasia esculenta (taro). Anti-cancer Drugs 23: 200-211, 2012.
2. Song G, Ohashi T, Sakamoto N and Sato M: Adhesive force of human hepatoma HepG2 cells to endothelial cells and expression of E-selectin. Mol Cell Biomech 3: 61-68, 2006.

3. Weber GF: Molecular mechanisms of metastasis. Cancer Lett 270: 181-190, 2008

4. Festuccia C, Bologna M, Gravina GL, Guerra F, Angelucci A, Villanova I, Millimaggi D and Teti A: Osteoblast conditioned media contain TGF-beta1 and modulate the migration of prostate tumor cells and their interactions with extracellular matrix components. Int J Cancer 81: 395-403, 1999.

5. Festuccia C, Guerra F, D'Ascenzo S, Giunciuglio D, Albini A and Bologna $\mathrm{M}$ : In vitro regulation of pericellular proteolysis in prostatic tumor cells treated with bombesin. Int J Cancer 75: 418-431, 1998.

6. Hu H, Ahn NS, Yang X,Lee YS and Kang KS: Ganoderma lucidum extract induces cell cycle arrest and apoptosis in MCF-7 human breast cancer cell. Int J Cancer 102: 250-253, 2002.

7. Lee SM, Li ML, Tse YC, et al: Paeoniae Radix, a Chinese herbal extract, inhibit hepatoma cells growth by inducing apoptosis in a p53 independent pathway. Life Sci 71: 2267-2277, 2002.

8. Cheng YL, Lee SC, Lin SZ, Chang WL, Chen YL, Tsai NM, Liu YC, Tzao C, Yu DS and Harn HJ: Anti-proliferative activity of Bupleurum scrozonerifolium in A549 human lung cancer cells in vitro and in vivo. Cancer Lett 222:183-193, 2005.

9. Wang XF, Du J, Zhang TL, Zhou QM, Lu YY, Zhang H and Su SB. Inhibitory effects of PC-SPESII herbal extract on human breast cancer metastasis. Evid Based Complement Alternat Med 2013: 894386, 2013.

10. Shimizu T, Torres MP, Chakraborty S, Souchek JJ, Rachagani S, Kaur S, Macha M, Ganti AK, Hauke RJ and Batra SK: Holy Basil leaf extract decreases tumorigenicity and metastasis of aggressive human pancreatic cancer cells in vitro and in vivo: potential role in therapy. Cancer Lett 336: 270-280, 2013.

11. Health Department and National Chinese Medicine Management Office: State Administration of Traditional Chinese Medicine of People's Republic of China. Zhong-hua-ben-cao, Shanghai Science and Technology Publisher, Shanghai, China, pp157-160, 1999.

12. Li YL, Gan GP, Zhang HZ, Wu HZ, Li CL, Huang YP, Liu YW and Liu JW: A flavonoid glycoside isolated from Smilax china L. rhizome in vitro anticancer effects on human cancer cell lines. J Ethnopharmacol 113: 115-124, 2007.

13. Shu XS, Gao ZH and Yang XL: Anti-inflammatory and anti-nociceptive activities of Smilax china L. aqueous extract. J Ethnopharmacol 103: 327-332, 2006.

14. Koressaar T and Remm M: Enhancements and modifications of primer design program Primer3. Bioinformatics 23: 1289-1291, 2007.

15. Kim MS, You MK, Rhuy DY, Kim YJ, Baek HY and Kim HA: Loquat (Eriobotrya japonica) extracts suppress the adhesion, migration and invasion of human breast cancer cell line. Nutr Res Pract 3: 259-264, 2009.

16. Bogenrieder T and Herlyn M: Axis of evil: molecular mechanisms of cancer metastasis. Oncogene 22: 6524-6536, 2003.

17. Du J, Wang XF, Zhou QM, Zhang TL, Lu YY, Zhang H and Su SB: Evodiamine induces apoptosis and inhibits metastasis in MDA-MB-231 human breast cancer cells in vitro and in vivo. Oncol Rep 30: 685-694, 2013.

18. Andres SA, Edwards AB and Wittliff JL: Expression of urokinase-type plasminogen activator (uPA), its receptor (uPAR), and inhibitor (PAI-1) in human breast carcinomas and their clinical relevance. J Clin Lab Anal 26: 93-103, 2012.

19. Dan K, Behrendt N, Brunner N, Ellis V, Ploug M and Pyke C: The urokinase receptor. Protein structure and role in plasminogen activation and cancer invasion. Fibrinolysis 8 (Suppl 1): 189-203, 1994.

20. Brinckerhoff CE and Matrisian LM: Matrix metalloproteinases: a tail of a frog that became a prince. Nat Rev Mol Cell Biol 3: 207-214, 2002.

21. Figueira RC, Gomes LR, Neto JS, Silva FC, Silva ID and Sogayar MC: Correlation between MMPs and their inhibitors in breast cancer tumor tissue specimens and in cell lines with different metastatic potential. BMC Cancer 9: 20, 2009.

22. Liotta LA and Stetler-Stevenson WG: Tumor invasion and metastasis: an imbalance of positive and negative regulation. Cancer Res 51 (18 Suppl): 5054s-5059s, 1991.

23. Albini A, Melchiori A, Santi L, Liotta LA, Brown PD and Stetler-Stevenson WG: Tumor cell invasion inhibited by TIMP-2. J Natl Cancer Inst 83: 775-779, 1991. 